\title{
A Mixed Volume Element With Characteristic Mixed Volume Element for Contamination Transport Problem
}

\author{
Yirang Yuan ${ }^{1}$, Changfeng $\mathrm{Li}^{2}$, Huailing Song ${ }^{3} \&$ Tongjun Sun ${ }^{1}$ \\ ${ }^{1}$ Institute of Mathematics, Shandong University, Jinan, China \\ ${ }^{2}$ School of Economics, Shandong University, Jinan, China \\ ${ }^{3}$ School of Mathematics and Econometrics, Hunan University, Changsha, China \\ Correspondence: Yirang Yuan, Institute of Mathematics, Shandong University, Shanda Nanlu No. 27, Jinan, 250100, \\ China. E-mail: yryuan@sdu.edu.cn
}

Received: May 20, 2020 Accepted: July 6, 2020 Online Published: August 19, 2020

doi:10.5539/jmr.v12n5p1 URL: https://doi.org/10.5539/jmr.v12n5p1

\begin{abstract}
Nonlinear systems of convection-dominated diffusion equations are used as the mathematical model of contamination transport problem which is an important topic in environ mental protection science. An elliptic equation defines the pressure, a convection-diffusion equation expresses the concentration of contamination, and an ordinary differential equation interprets the surface absorption concentration. The transport pressure appears in the equation of the concentration which determines the Darcy velocity and also controls the physical process. The method of conservative mixed volume element is used to solve the flow equation which improves the computational accuracy of Darcy velocity by one order. We use the mixed volume element with the characteristic to approximate the concentration. This method of characteristic not only preserves the strong computational stability at sharp front, but also eliminates numerical dispersion and nonphysical oscillation. In the present scheme, we could adopt a large step without losing accuracy. The diffusion is approximated by the mixed volume element. The concentration and its adjoint vector function are obtained simultaneously, and the locally conservative law is preserved. An optimal second order estimates in $l^{2}$-norm is derived.
\end{abstract}

Keywords: contamination transport, mixed finite volume element, characteristic mixed volume element, local conservation of mass, second order estimate in $l^{2}$ norm

\section{Introduction}

Numerical simulation of the contamination transport problem is an efficient way to find how to protect the environment and treat the pollution. The physical interpretation is given by a mathematical system of partial differential equations. In this model, an elliptic equation defines the pressure, a convection-diffusion equation expresses the concentration of contamination, and an ODE interprets the surface absorption concentration. We provide a simplified physical background in the following. When the chemical substances are dissolved in the underground water, the substances are absorbed by the surface of the media, and then the chemical reaction takes place. The chemical reaction also affects the transportation of the solutes as the underground water moves. Consideration of the reaction helps us to understand how the contamination transport in terms of time and space. The reaction speed depends on the absorption. The equilibrium is donoted quick reaction, and non-equilibrium absorption is denoted by slow reaction speed. In this paper, we discuss the non-equilibrium absorption. The following mathematical model is discussed with the initial-boundary value conditions (Dawson, Van Duiji \& Wheeler, 1994; Dawson, 1998; Hornung, 1988; Vogt, 1982):

$$
\begin{gathered}
\nabla \cdot \mathbf{u}=-\nabla \cdot(\kappa(c) \nabla p)=q(X, t), X=(x, y, z)^{T} \in \Omega, t \in J=(0, T], \\
\mathbf{u}=-\kappa(c) \nabla p, X \in \Omega, t \in J, \\
\theta(X) \frac{\partial c}{\partial t}+\rho(X) \frac{\partial s}{\partial t}+\nabla \cdot(\mathbf{u} c)-\nabla \cdot(\theta D \nabla c)=0, X \in \Omega, t \in J, \\
\frac{\partial s}{\partial t}=\hat{\alpha}(\phi(c)-s), X \in \Omega, t \in J,
\end{gathered}
$$

where $\Omega$ is a bounded domain in $\mathbb{R}^{3} \cdot p(X, t)$ denotes the pressure, and $\mathbf{u}=\left(u_{x}, u_{y}, u_{z}\right)^{T}$ is Darcy velocity. The functions $c(X, t)$ and $s(X, t)$ denote the contamination concentration and non-equilibrium absorption concentration. $\kappa(c)$ is the permeability. $\theta(X)$ and $\rho(X)$, two positive functions, are water storage rates of moving water and the density, respectively. $D$ 
is a total matrix of molecular diffusion and chemical dispersion,

$$
\theta D(X, \mathbf{u})=\theta D_{\mathrm{mol}}(X) \mathbf{I}+\alpha_{1}\left|\mathbf{u}^{\beta}\left(\begin{array}{ccc}
\hat{u}_{x}^{2} & \hat{u}_{x} \hat{u}_{y} & \hat{u}_{x} \hat{u}_{z} \\
\hat{u}_{y} \hat{u}_{x} & \hat{u}_{y}^{2} & \hat{u}_{y} \hat{u}_{z} \\
\hat{u}_{z} \hat{u}_{x} & \hat{u}_{z} \hat{u}_{y} & \hat{u}_{z}^{2}
\end{array}\right)+\alpha_{t}\right| \mathbf{u}^{\beta}\left(\begin{array}{ccc}
\hat{u}_{y}^{2}+\hat{u}_{z}^{2} & -\hat{u}_{x} \hat{u}_{y} & -\hat{u}_{x} \hat{u}_{z} \\
-\hat{u}_{x} \hat{u}_{y} & \hat{u}_{x}^{2}+\hat{u}_{z}^{2} & -\hat{u}_{y} \hat{u}_{z} \\
-\hat{u}_{x} \hat{u}_{z} & -\hat{u}_{z} \hat{u}_{y} & \hat{u}_{x}^{2}+\hat{u}_{y}^{2}
\end{array}\right) .
$$

$D_{\text {mol }}(X)$ is a molecular diffusion matrix and $\mathbf{I}$ is a $3 \times 3$ identity matrix. $\alpha_{l}$ and $\alpha_{t}$ respectively represent longitudinal and transverse diffusion coefficients. $\hat{u}_{x}, \hat{u}_{y}$ and $\hat{u}_{z}$ are three direction cosines of Darcy velocity $\mathbf{u}$. Generally speaking, $\beta$ is a constant not less than 2 , and diffusion-dispersion matrix is positive definite. Here we only consider the molecular diffusion. Suppose that $D_{*} \leq D_{\mathrm{mol}}(X) \leq D^{*}$ holds for positive constants $D_{*}$ and $D^{*}$. $\hat{\alpha}$, a positive constant, is the exchange coefficient. $\phi(c)$ denotes the isothermal absorption, generally defined by two different methods

(I) Langmuir isotherm, $\phi(c)=\frac{k_{1} c}{1+k_{2} c}, k_{1}, k_{2}>0$,

(II) Freundlinch isotherm, $\phi(c)=k_{3} c^{p}, k_{3}>0$, where $p \in[0,1]$.

The case (II) is a degraded parabolic equation for $p<1$, and $\phi \in C^{0} \cap C^{1}((-\infty, 0) \cup(0, \infty))$ is an increasing function with $\phi(0)=0$. Since the parameter $p \in(0,1)$ is locally Hölder continuous, thus the regularity of $c$ decreases. $q(X, t)$ is the source and sink term. Three unknown functions are $p(X, t), c(X, t)$ and $s(X, t)$.

Assume that the mixture is impermeable across the boundary,

$$
\mathbf{u} \cdot v=0, X \in \partial \Omega,(\theta D \nabla c-c \mathbf{u}) \cdot v=0, X \in \partial \Omega, t \in J,
$$

where $v$ denotes the outer normal vector at the boundary $\partial \Omega$ of $\Omega$.

The initial conditions are defined by

$$
c(X, 0)=c_{0}(X), s(X, 0)=s_{0}(X), X \in \Omega .
$$

The following restriction is introduced to confirm the existence and uniqueness

$$
\int_{\Omega} q(X, t) d X=0, \int_{\Omega} p(X, t) d X=0, t \in J
$$

The Lipschitz continuity does not hold for this physical model, and this nonlinearity decreases the regularity. It brings trouble on numerical analysis for the full discrete scheme. Dawson first presents and analyzes the method of characteristicsGalerkin for non-equilibrium absorption (Dawson, Van Duiji \& Wheeler, 1994; Dawson, 1998). While numerical oscillation possibly appears and the local conservation of mass is lost. Furthermore, an upwind with mixed finite element method is put forward to discuss a nonlinear contamination transport equation with equilibrium absorption in one variable. Convergence analysis is shown only for a local low-order semidiscrete algorithm. It is well-known that standard finite element could not solve convection-diffusion problems well because of strong numerical oscillation. In order to treat this problem well, some efficient numerical methods are put forward such as characteristic difference, characteristic finite element (Ewing, 1983; Yuan, 1996,1999,2013), upstream-weighted finite difference (Todd, ODell \& Hirasaki, 1972), high-order Godunov scheme (Bell, Dawson \& Shubin, 1988), streamline diffusion method (Johnson, 1986), least squares mixed finite element (Yang, 2000), modified method of characteristics with Galerkin finite element (MMOC-Galerkin) (Dawson, Russell \& Wheeler, 1989), Eulerian-Lagrangian localized adjoint method (ELLAM) ( Cella, Russell, Herrera \& Ewing, 1990) and so on. This problem should consider an important physical conservation. Arbogast and Wheeler present a type of characteristic mixed finite element (Arbogast \& Wheeler, 1995) to solve the convection-diffusion equation efficiently, where MMOC-Galerkin is conservative locally on every elements and a $3 / 2$ order error estimates is derived. Lots of mapping integrals of test functions make the computations more complicated. Therefore, we develop and improve substantially the work of Arbogast and Wheeler (Arbogast \& Wheeler, 1995), and put forward a mixed finite element-characteristic mixed element method. This method decreases computational work greatly, and the feasibility and effectiveness are tested experimentally (Sun \& Yuan, 2009). This method could not be generalized for three-dimensional problems. Finite volume method (Cai, 1991; Li \& Chen, 1994) is carried out simply, and it has high accuracy and conservation. Thus it is an effective tool for solving partial differential equations. The method of mixed finite element (Douglas, Ewing \& Wheeler, 1983, 19832; Raviart \& Thomas, 1977) can solve the pressure and Darcy velocity simultaneously, and develops the accuracy. Combined the above two methods, a mixed finite volume method is discussed (Russell, 1995; Vogt, 1982; Weiser \& Wheeler, 1988) and numerical experiments are argued (Cai, Jones, Mccormilk \& Russell, 1997; Jones, 1995). Theoretical analysis is given for elliptic problem and a general discussion frame is given for mixed finite volume method (Chou, Kawk \& Vassileviki, 1998, 2000; Chou \& Vassileviki, 1999). Rui and Pan use this method to discuss 
numerical computation for hypotonic oil-gas flow problems (Pan \& Rui, 2012, 20122). In this paper, a type of mixed volume element with characteristic mixed volume element method is put forward for three-dimensional underground water pollution problem. The pressure and Darcy velocity are computed simultaneously by a conservative mixed volume element and the accuracy of Darcy velocity is improved. A conservative characteristic mixed finite volume element is used to compute the concentration, where the convection is approximated along the characteristics and the diffusion is discretized by the mixed volume element. The method of characteristics obtains the values at the sharp fronts very well. In actual computations, a large time step may be adopted and the efficiency is improved. Piecewise-defined constants are taken as test functions, and the local conservation of mass is preserved. Finally, we obtain an optimal order estimates in $L^{2}$ norm superior to 3/2-order (Arbogast \& Wheeler, 1995). Thus, the potential is shown to be an efficient tool in solving some actual applications (Arbogast \& Wheeler, 1995; Ewing, 1983; Shen, Liu \& Tang, 2002; Yuan, 2013).

Some symbols and assumptions are introduced. The regularity conditions of (1)-(7) are defined by

$$
\left\{\begin{array}{l}
p \in L^{\infty}\left(H^{1}\right), \\
\mathbf{u} \in L^{\infty}\left(H^{1}(\mathrm{div})\right) \cap L^{\infty}\left(W_{\infty}^{1}\right) \cap W_{\infty}^{1}\left(L^{\infty}\right) \cap H^{2}\left(L^{2}\right), \\
c \in L^{\infty}\left(H^{2}\right) \cap H^{1}\left(H^{1}\right) \cap L^{\infty}\left(W_{\infty}^{1}\right) \cap H^{2}\left(L^{2}\right), \\
s \in L^{\infty}\left(H^{2}\right) \cap H^{2}\left(L^{2}\right) .
\end{array}\right.
$$

Suppose that the coefficients satisfy the following positive definite conditions

$$
\text { (C) } 0<k_{*} \leq \kappa(c) \leq k^{*}, 0<\theta_{*} \leq \theta(X) \leq \theta^{*}, 0<\rho_{*} \leq \rho(X) \leq \rho^{*}, 0<D_{*} \leq D(X) \leq D^{*},
$$

where $k_{*}, k^{*}, \theta_{*}, \theta^{*}, \rho_{*}, \rho^{*}, D_{*}$ and $D^{*}$ are positive constants.

For simplicity, the problem (1)-(7) is assumed to be $\Omega$-periodic (Dawson, Van Duiji \& Wheeler, 1994; Dawson, 1998; Ewing, 1983; Hornung, 1988; Vogt, 1982), that is, all the functions are $\Omega$-periodic. This assumption seems physically reasonable, since no-flow boundaries are generally treated by reflection, and because interior flow factors are much more important than boundary effects. Thus, the conditions (5) possibly is dropped (Ewing, 1983; Yuan, 1999, 2013).

In this paper, let $K$ and $\varepsilon$ denote a generic positive constant and a generic small positive number, respectively. They may have different definitions at different places.

\section{The Preparations}

Two different partitions are supposed to be regular for showing the numerical composite scheme. The first partition with a large step is nonuniform for solving the pressure and Darcy velocity, and the second nonuniform one is for the concentration. For simplicity, take $\Omega=\{[0,1]\}^{3}$ and let $\partial \Omega$ denote the boundary. The first partition is defined by $\delta_{x} \times \delta_{y} \times \delta_{z}$,

$$
\begin{aligned}
& \delta_{x}: 0=x_{1 / 2}<x_{3 / 2}<\cdots<x_{N_{x}-1 / 2}<x_{N_{x}+1 / 2}=1, \\
& \delta_{y}: 0=y_{1 / 2}<y_{3 / 2}<\cdots<y_{N_{y}-1 / 2}<y_{N_{y}+1 / 2}=1, \\
& \delta_{z}: 0=z_{1 / 2}<z_{3 / 2}<\cdots<z_{N_{z}-1 / 2}<z_{N_{z}+1 / 2}=1 .
\end{aligned}
$$

$N_{x}, N_{y}$ and $N_{z}$ are positive integers. The element is defined by $\Omega_{i j k}=\left\{(x, y, z): x_{i-1 / 2}<x<x_{i+1 / 2}, y_{j-1 / 2}<y<\right.$ $\left.y_{j+1 / 2}, z_{k-1 / 2}<z<z_{k+1 / 2}\right\} . x_{i}, y_{j}, z_{k}, h_{x_{i}}, h_{y_{j}}, h_{z k}, h_{x, i+1 / 2}, h_{y, j+1 / 2}, h_{z, k+1 / 2}$ are defined in the traditional manner (Weiser \& Wheeler, 1988; Yuan, 2013). Let $h_{x}=\max _{1 \leq i \leq N_{x}}\left\{h_{x_{i}}\right\}, h_{y}=\max _{1 \leq j \leq N_{y}}\left\{h_{y_{j}}\right\}, h_{z}=\max _{1 \leq k \leq N_{z}}\left\{h_{z_{k}}\right\}$ and $h_{p}=\left(h_{x}^{2}+h_{y}^{2}+h_{z}^{2}\right)^{1 / 2}$. The spaces are defined by $S_{h}=M_{-1}^{0}\left(\delta_{x}\right) \otimes M_{-1}^{0}\left(\delta_{y}\right) \otimes M_{-1}^{0}\left(\delta_{z}\right), V_{h}=\left\{\mathbf{w} \mid \mathbf{w}=\left(w^{x}, w^{y}, w^{z}\right), w^{x} \in M_{0}^{1}\left(\delta_{x}\right) \otimes M_{-1}^{0}\left(\delta_{y}\right) \otimes M_{-1}^{0}\left(\delta_{z}\right), w^{y} \in\right.$ $\left.M_{-1}^{0}\left(\delta_{x}\right) \otimes M_{0}^{1}\left(\delta_{y}\right) \bigotimes M_{-1}^{0}\left(\delta_{z}\right), w^{z} \in M_{-1}^{0}\left(\delta_{x}\right) \otimes M_{-1}^{0}\left(\delta_{y}\right) \bigotimes M_{0}^{1}\left(\delta_{z}\right),\left.\mathbf{w} \cdot \gamma\right|_{\partial \Omega}=0\right\}$.

The inner products and norms are defined as follows

$$
\begin{aligned}
& (v, w)_{m}=\sum_{i=1}^{N_{x}} \sum_{j=1}^{N_{y}} \sum_{k=1}^{N_{z}} h_{x_{i}} h_{y_{j}} h_{z z_{k}} v_{i j k} w_{i j k},(v, w)_{x}=\sum_{i=1}^{N_{x}} \sum_{j=1}^{N_{y}} \sum_{k=1}^{N_{z}} h_{x_{i-1 / 2}} h_{y_{j}} h_{z k} v_{i-1 / 2, j k} w_{i-1 / 2, j k}, \\
& (v, w)_{y}=\sum_{i=1}^{N_{x}} \sum_{j=1}^{N_{y}} \sum_{k=1}^{N_{z}} h_{x_{i}} h_{y_{j-1 / 2}} h_{z k} v_{i, j-1 / 2, k} w_{i, j-1 / 2, k}, \\
& (v, w)_{z}=\sum_{i=1}^{N_{x}} \sum_{j=1}^{N_{y}} \sum_{k=1}^{N_{z}} h_{x_{i}} h_{y_{j}} h_{z k-1 / 2} v_{i j, k-1 / 2} w_{i j, k-1 / 2}, \\
& \|v\|_{s}^{2}=(v, v)_{s}, s=m, x, y, z, \quad\|v\|_{\infty}=\max _{1 \leq i \leq N_{x}, 1 \leq j \leq N_{y}, 1 \leq k \leq N_{z}}\left|v_{i j k}\right|, \\
& \|v\|_{\infty(x)}=\max _{1 \leq i \leq N_{x}, 1 \leq j \leq N_{y}, 1 \leq k \leq N_{z}}\left|v_{i-1 / 2, j k}\right|, \quad\|v\|_{\infty(y)}=\underset{1 \leq i \leq N_{x}, 1 \leq j \leq N_{y}, 1 \leq k \leq N_{z}}{=}\left|v_{i, j-1 / 2, k}\right|,
\end{aligned}
$$


$\|v\|_{\infty(z)}=\max _{1 \leq i \leq N_{x}, 1 \leq j \leq N_{y}, 1 \leq k \leq N_{z}}\left|v_{i j, k-1 / 2}\right|$.

For a vector $\mathbf{w}=\left(w^{x}, w^{y}, w^{z}\right)^{T}$, let

$\left\|\left|\mathbf{w}\left\|=\left(\left\|w^{x}\right\|_{x}^{2}+\left\|w^{y}\right\|_{y}^{2}+\left\|w^{z}\right\|_{z}^{2}\right)^{1 / 2},\right\|\right| \mathbf{w}\right\|_{\infty}=\left\|w^{x}\right\|_{\infty(x)}+\left\|w^{y}\right\|_{\infty(y)}+\left\|w^{z}\right\|_{\infty(z)}$,

$\|\mathbf{w}\|_{m}=\left(\left\|w^{x}\right\|_{m}^{2}+\left\|w^{y}\right\|_{m}^{2}+\left\|w^{z}\right\|_{m}^{2}\right)^{1 / 2},\|\mathbf{w}\|_{\infty}=\left\|w^{x}\right\|_{\infty}+\left\|w^{y}\right\|_{\infty}+\left\|w^{z}\right\|_{\infty}$.

The difference operators and other notation are introduced,

$$
\begin{gathered}
{\left[d_{x} v\right]_{i+1 / 2, j k}=\frac{v_{i+1, j k}-v_{i j k}}{h_{x, i+1 / 2}},\left[d_{y} v\right]_{i, j+1 / 2, k}=\frac{v_{i, j+1, k}-v_{i j k}}{h_{y, j+1 / 2}},\left[d_{z} v\right]_{i j, k+1 / 2}=\frac{v_{i j, k+1}-v_{i j k}}{h_{z, k+1 / 2}} ;} \\
{\left[D_{x} w\right]_{i j k}=\frac{w_{i+1 / 2, j k}-w_{i-1 / 2, j k}}{h_{x_{i}}},\left[D_{y} w\right]_{i j k}=\frac{w_{i, j+1 / 2, k}-w_{i, j-1 / 2, k}}{h_{y_{j}}},\left[D_{z} w\right]_{i j k}=\frac{w_{i j, k+1 / 2}-w_{i j, k-1 / 2}}{h_{z k}} ;} \\
\hat{w}_{i j k}^{x}=\frac{w_{i+1 / 2, j k}^{x}+w_{i-1 / 2, j k}^{x}}{2}, \hat{w}_{i j k}^{y}=\frac{w_{i, j+1 / 2, k}^{y}+w_{i, j-1 / 2, k}^{y}}{2}, \hat{w}_{i j k}^{z}=\frac{w_{i j, k+1 / 2}^{z}+w_{i j, k-1 / 2}^{z}}{2}, \\
\bar{w}_{i j k}^{x}=\frac{h_{x, i+1}}{2 h_{x, i+1 / 2}} w_{i j k}+\frac{h_{x, i}}{2 h_{x, i+1 / 2}} w_{i+1, j k}, \bar{w}_{i j k}^{y}=\frac{h_{y, j+1}}{2 h_{y, j+1 / 2}} w_{i j k}+\frac{h_{y, j}}{2 h_{y, j+1 / 2}} w_{i, j+1, k}, \\
\bar{w}_{i j k}^{z}=\frac{h_{z, k+1}}{2 h_{z, k+1 / 2}} w_{i j k}+\frac{h_{z, k}}{2 h_{z, k+1 / 2}} w_{i j, k+1},
\end{gathered}
$$

and $\hat{\mathbf{w}}_{i j k}=\left(\hat{w}_{i j k}^{x}, \hat{w}_{i j k}^{y}, \hat{w}_{i j k}^{z}\right)^{T}, \overline{\mathbf{w}}_{i j k}=\left(\bar{w}_{i j k}^{x}, \bar{w}_{i j k}^{y}, \bar{w}_{i j k}^{z}\right)^{T}$.

The properties are prepared for the following numerical analysis.

Lemma 1 For $v \in S_{h}, \mathbf{w} \in V_{h}$,

$$
\left(v, D_{x} w^{x}\right)_{m}=-\left(d_{x} v, w^{x}\right)_{x},\left(v, D_{y} w^{y}\right)_{m}=-\left(d_{y} v, w^{y}\right)_{y},\left(v, D_{z} w^{z}\right)_{m}=-\left(d_{z} v, w^{z}\right)_{z} .
$$

Lemma 2 For $\mathbf{w} \in V_{h}$,

$$
\|\hat{\mathbf{w}}\|_{m} \leq\|\mid \mathbf{w}\| .
$$

Lemma 3 For $q \in S_{h}$,

$$
\left\|\bar{q}^{x}\right\|_{x} \leq M\|q\|_{m},\left\|\bar{q}^{y}\right\|_{y} \leq M\|q\|_{m},\left\|\bar{q}^{z}\right\|_{z} \leq M\|q\|_{m},
$$

where $M$ is a constant independent of $q$ and $h$.

Lemma 4 For $\mathbf{w} \in V_{h}$,

$$
\left\|w^{x}\right\|_{x} \leq\left\|D_{x} w^{x}\right\|_{m},\left\|w^{y}\right\|_{y} \leq\left\|D_{y} w^{y}\right\|_{m},\left\|w^{z}\right\|_{z} \leq\left\|D_{z} w^{z}\right\|_{m} .
$$

Another partition is obtained by refining the coarse partition of $\Omega=\{[0,1]\}^{3}$ uniformly. Generally, we take the step by $h_{c}=h_{p} / 2$ or $h_{p} / 4$.

Since the absorption concentration changes slow and stably, eq. (2) is discussed on the first partition. A first-order finite element space $M_{h}$ is constructed on a hexahedron element $\Omega_{i j k}=\left[x_{i-1 / 2}, x_{i+1 / 2}\right] \times\left[y_{j-1 / 2}, y_{j+1 / 2}\right] \times\left[z_{k-1 / 2}, z_{k+1 / 2}\right]$ (Cialet, 1978; Jiang \& Pang, 1979).

\section{The Procedures of Mixed Finite Volume Element With Modified Characteristic Mixed Volume Element}

\subsection{The Procedures}

In order to use the mixed finite volume element, we rewrite eq.(1) in a normalized formulation

$$
\begin{aligned}
& \nabla \cdot \mathbf{u}=q, \\
& \mathbf{u}=-k(c) \nabla p .
\end{aligned}
$$

For eq.(2), considering that the flow transports along the characteristic direction, thus we apply the method of characteristics to approximate the first-order hyperbolic term. The computational algorithm could use a large time step and has 
the strong stability and high order accuracy. Let $\psi(X, \mathbf{u})=\left[\theta^{2}(X)+|\mathbf{u}|^{2}\right]^{1 / 2}, \frac{\partial}{\partial \tau}=\psi^{-1}\left\{\theta \frac{\partial}{\partial t}+\mathbf{u} \cdot \nabla\right\}$. Eq.(2) is rewritten as follows for applying the method of mixed volume element,

$$
\begin{aligned}
& \psi \frac{\partial c}{\partial \tau}+\rho \frac{\partial s}{\partial t}+\nabla \cdot \mathbf{g}=f(X, c), \\
& \mathbf{g}=-\theta D \nabla c
\end{aligned}
$$

where $f(X, c)=-q c$.

Let $P, \mathbf{U}, C, \mathbf{G}$ and $S$ denote the discrete solutions of $p, \mathbf{u}, c, \mathbf{g}$ and $s$, respectively. The pressure and Darcy velocity are computed by the method of characteristic mixed volume element,

$$
\begin{aligned}
& \left(D_{x} U^{x, n+1}+D_{y} U^{y, n+1}+D_{z} U^{z, n+1}, v\right)_{m}=\left(q^{n+1}, v\right)_{m}, \forall v \in S_{h}, \\
& \left(k^{-1}\left(\bar{C}^{x, n}\right) U^{x, n+1}, w^{x}\right)_{x}+\left(k^{-1}\left(\bar{C}^{y, n}\right) U^{y, n+1}, w^{y}\right)_{y}+\left(k^{-1}\left(\bar{C}^{z, n}\right) U^{z, n+1}, w^{z}\right)_{z} \\
& -\left(P^{n+1}, D_{x} w^{x}+D_{x} w^{y}+D_{x} w^{z}\right)_{m}=0, \forall \mathbf{w} \in V_{h} .
\end{aligned}
$$

The derivative along the characteristics in (13a) is approximated by the backward difference quotient

$$
\frac{\partial c^{n+1}}{\partial \tau} \approx \frac{c^{n+1}-c^{n}\left(X-\theta^{-1} \mathbf{u}^{n+1}(X) \Delta t\right)}{\Delta t\left(1+\theta^{-2}\left|\mathbf{u}^{n+1}\right|^{2}\right)^{1 / 2}} .
$$

Eq.(13) is discretized by the characteristic mixed volume element method

$$
\begin{aligned}
& \left(\theta \frac{C^{n+1}-\hat{C}^{n}}{\Delta t}, v\right)_{m}+\left(\rho \frac{S^{n+1}-S^{n}}{\Delta t}, v\right)_{m}+\left(D_{x} G^{x, n+1}+D_{y} G^{y, n+1}+D_{z} G^{z, n+1}, v\right)_{m} \\
& =\left(f\left(\hat{C}^{n}\right), v\right)_{m}, \forall v \in S_{h}, \\
& \left.\left(D^{-1} G^{x, n+1}, w^{x}\right)_{x}+\left(D^{-1} G^{y, n+1}, w^{y}\right)_{y}+\left(D^{-1} G^{z, n+1}\right), w^{z}\right)_{z}-\left(C^{n+1}, D_{x} w^{x}+D_{y} w^{y}+D_{z} w^{z}\right)_{m} \\
& =0, \forall \mathbf{w} \in V_{h},
\end{aligned}
$$

where $\hat{C}^{n}=C^{n}\left(\hat{X}^{n}\right), \hat{X}^{n}=X-\theta^{-1} \mathbf{U}^{n+1} \Delta t$.

The adsorption concentration changes slow and stably with respect to $t$, so we obtain an explicit scheme to approximate eq.(3),

$$
\left(\frac{S^{n+1}-S^{n}}{\Delta t}, \varphi\right)=\hat{\alpha}\left(\phi\left(C^{n}\right)-S^{n}, \varphi\right), \quad \varphi \in M_{h}
$$

Initial approximations are defined by

$$
C^{0}=\tilde{C}^{0}, \mathbf{G}^{0}=\tilde{\mathbf{G}}^{0}, S^{0}=\tilde{S}^{0}, X \in \Omega,
$$

where $\left(\tilde{C}^{0}, \tilde{\mathbf{G}}^{0}\right)$ is the elliptic projection of $\left(c_{0}, \mathbf{g}_{0}\right)$, and $\tilde{S}^{0}$ is an $L^{2}$ projection of $s_{0}$ (see the definitions in the next section).

The composite procedures run as follows. First of all, the elliptic projection and the initial values are used to obtain $\left\{\tilde{C}^{0}, \tilde{\mathbf{G}}^{0}\right\}$, as the initial approximations of $c_{0}$ and $\mathbf{g}_{0}=-\theta D \nabla c_{0}$, i.e. $C^{0}=\tilde{C}^{0}, \mathbf{G}^{0}=\tilde{\mathbf{G}}^{0}$. Use $s_{0}$ and the $L^{2}$ projection to get $S^{0}=\tilde{S}^{0}$. Secondly, $S^{1}$ is computed by (16). Then, $\left\{\mathbf{U}^{1}, P^{1}\right\}$ is computed by the method of conjugate gradient and mixed volume element (14). Similarly, $\left\{C^{1}, \mathbf{G}^{1}\right\}$ is obtained by (15). Next, $S^{2},\left\{\mathbf{U}^{2}, P^{2}\right\}$ and $\left\{C^{2}, \mathbf{G}^{2}\right\}$ are computed by (15), (14) and (16). The computations proceed repeatedly and all the numerical solutions are obtained. By the positive definite condition (C), the solutions exist and are unique.

\subsection{The Conservation of Mass}

Assume that the problem (1)-(7) has no source or sink, i.e., $q \equiv 0$, and assume that the boundary condition is impermeable. Here we ignore the effect of adsorption. Thus, for simplicity we take $l=1$, or suppose that two partitions are the same. On every element $J_{c} \subset \Omega, J_{c}=\Omega_{i j k}=\left[x_{i-1 / 2}, x_{i+1 / 2}\right] \times\left[y_{j-1 / 2}, y_{j+1 / 2}\right] \times\left[z_{k-1 / 2}, z_{k+1 / 2}\right]$, the local conservation of mass is interpreted for eq.(2) by

$$
\int_{J_{c}} \psi \frac{\partial c}{\partial \tau} d X-\int_{\partial J_{c}} \mathbf{g} \cdot \gamma_{J_{c}} d S=0
$$

$\partial J_{c}$ denotes the boundary of $J_{c}$ and $\gamma_{J_{c}}$ denotes its outer normal vector. Then, the discrete local conservation holds for eq.(15a). 
Theorem 1. If $q \equiv 0$, and the factor of surface absorption is ignored, then on every element $J_{c} \subset \Omega$, (15a) satisfies the conservation law

$$
\int_{J_{c}} \theta \frac{C^{n+1}-\hat{C}^{n}}{\Delta t} d X-\int_{\partial J_{c}} \mathbf{G}^{n+1} \cdot \gamma_{J_{c}} d S=0 .
$$

Proof. Note that $v=\left\{\begin{array}{ll}1, & J_{c}=\Omega_{i j k}, \\ 0, & \text { otherwise, }\end{array} \quad v \in S_{h}\right.$. Then, (15a) turns into

$$
\left(\theta \frac{C^{n+1}-\hat{C}^{n}}{\Delta t}, 1\right)_{\Omega_{i j k}}+\left(D_{x} G^{x, n+1}+D_{y} G^{y, n+1}+D_{z} G^{z, n+1}, 1\right)_{\Omega_{i j k}}=0 .
$$

Using the previous notation, we have

$$
\begin{aligned}
& \left(\theta \frac{C^{n+1}-\hat{C}^{n}}{\Delta t}, 1\right)_{\Omega_{i j k}}=\theta_{i j k}\left(\frac{C_{i j k}^{n+1}-\hat{C}_{i j k}^{n}}{\Delta t}\right) h_{x_{i}} h_{y_{j}} h_{z k}=\int_{\Omega_{i j k}} \theta \frac{C^{n+1}-\hat{C}^{n}}{\Delta t} d X, \\
& \left(D_{x} G^{x, n+1}+D_{y} G^{y, n+1}+D_{z} G^{z, n+1}, 1\right)_{\Omega_{i j k}}=\left(G_{i+1 / 2, j k}^{x, n+1}-G_{i-1 / 2, j k}^{x, n+1}\right) h_{y_{j}} h_{z_{k}} \\
& +\left(G_{i, j+1 / 2, k}^{y, n+1}-G_{i, j-1 / 2, k}^{y, n+1}\right) h_{x_{i}} h_{z_{k}}+\left(G_{i j, k+1 / 2}^{z, n+1}-G_{i j, k-1 / 2}^{z, n+1}\right) h_{x_{i}} h_{y_{j}}=-\int_{\partial \Omega_{i j k}} \mathbf{G}^{n+1} \cdot \gamma_{J_{c}} d S .
\end{aligned}
$$

Substituting (21) into (20), we could complete this proof.

Furthermore, we get the conservation of mass on the whole domain.

Theorem 2. Suppose $q \equiv 0$ and the boundary is impermeable. The factor of surface absorption is ignored. Then,

$$
\int_{\Omega} \theta \frac{C^{n+1}-\hat{C}^{n}}{\Delta t} d X=0, n \geq 0
$$

Proof. Summing (19) on all the elements, we have

$$
\sum_{i, j, k} \int_{\Omega_{i j k}} \theta \frac{C^{n+1}-\hat{C}^{n}}{\Delta t} d X-\sum_{i, j, k} \int_{\partial \Omega_{i j k}} \mathbf{G}^{n+1} \cdot \gamma_{J_{c}} d S=0 .
$$

Then, the proof is completed by using $-\sum_{i, j, k} \int_{\partial \Omega_{i j k}} \mathbf{G}^{n+1} \cdot \gamma_{J_{c}} d S=-\int_{\partial \Omega} \mathbf{G}^{n+1} \cdot \gamma d S=0$.

\section{Convergence Analysis}

The elliptic projections are introduced first. $\tilde{\mathbf{U}} \in V_{h}$ and $\tilde{P} \in S_{h}$ are defined by

$$
\begin{aligned}
& \left(D_{x} \tilde{U}^{x}+D_{y} \tilde{U}^{y}+D_{z} \tilde{U}^{z}, v\right)_{m}=(q, v)_{m}, \forall v \in S_{h}, \\
& \left(k^{-1}(c) \tilde{U}^{x}, w^{x}\right)_{x}+\left(k^{-1}(c) \tilde{U}^{y}, w^{y}\right)_{y}+\left(k^{-1}(c) \tilde{U}^{z}, w^{z}\right)_{z}-\left(\tilde{P}, D_{x} w^{x}+D_{y} w^{y}+D_{z} w^{z}\right)_{m}=0, \forall \mathbf{w} \in V_{h},
\end{aligned}
$$

where $c$ denotes the exact solution of (1)-(7).

Let $F=f-\psi \frac{\partial c}{\partial \tau}-\rho \frac{\partial s}{\partial t}$. Define $\tilde{\mathbf{G}} \in V_{h}, \tilde{C} \in S_{h}$ by

$$
\begin{aligned}
& \left(D_{x} \tilde{G}^{x}+D_{y} \tilde{G}^{y}+D_{z} \tilde{G}^{z}, v\right)_{m}=(F, v)_{m}, \forall v \in S_{h}, \\
& \left(D^{-1} \tilde{G}^{x}, w^{x}\right)_{x}+\left(D^{-1} \tilde{G}^{y}, w^{y}\right)_{y}+\left(D^{-1} \tilde{G}^{z}, w^{z}\right)_{z}-\left(\tilde{C}, D_{x} w^{x}+D_{y} w^{y}+D_{z} w^{z}\right)_{m}=0, \forall \mathbf{w} \in V_{h} .
\end{aligned}
$$

The $L^{2}$ finite element projection $\tilde{S} \in M_{h}$ is defined by

$$
(S, z)=(s, z), \quad \forall z \in M_{h}
$$

Let $\pi=P-\tilde{P}, \eta=\tilde{P}-p, \sigma=\mathbf{U}-\tilde{\mathbf{U}}, \rho=\tilde{\mathbf{U}}-\mathbf{u}, \xi=C-\tilde{C}, \zeta=\tilde{C}-c, \alpha=\mathbf{G}-\tilde{\mathbf{G}}, \beta=\tilde{\mathbf{G}}-\mathbf{g}, \mu=S-\tilde{S}, \lambda=\tilde{S}-s$. Suppose that (1)-(7) satisfies the positive definite condition (C), and suppose that exact solutions satisfy the regularity (R). From the discussions of Weiser and Wheeler (Weiser \& Wheeler, 1988), we see that auxiliary functions $\{\tilde{\mathbf{U}}, \tilde{P}, \tilde{\mathbf{G}}, \tilde{C}\}$ of (24) and (25) exist and are unique. 
Lemma 5 If the coefficients and exact solutions of (1)-(7) satisfy (C) and (R), then there exist two constants $\bar{C}_{1}>0$ and $\bar{C}_{2}>0$ independent of $h$ such that

$$
\begin{aligned}
& \|\eta\|_{m}+\|\zeta\|_{m}+\|\mid \rho\|\|+\| \beta\|\|+\left\|\frac{\partial \eta}{\partial t}\right\|_{m}+\left\|\frac{\partial \zeta}{\partial t}\right\|_{m} \leq \bar{C}_{1}\left\{h_{p}^{2}+h_{c}^{2}\right\} \\
& \|\tilde{\mathbf{U}}\|_{\infty}+\|\tilde{\mathbf{G}}\|_{\infty} \leq \bar{C}_{2}
\end{aligned}
$$

Under the same assumptions, using the property of $L^{2}$ projection (Cialet, 1978; Jiang \& Pang, 1979), we have the following estimates.

Lemma 6 Suppose that the adsorption concentration satisfies the regularity (R). Then there exists a constant $\bar{C}_{3}>0$ independent of $h_{c}$ such that

$$
\|\lambda\|_{L^{2}}+\left\|\frac{\partial \lambda}{\partial t}\right\|_{L^{2}} \leq \bar{C}_{3} h_{c}^{2}
$$

$\pi$ and $\sigma$ are estimated first. Subtracting (24a) $\left(t=t^{n+1}\right)$ and (24b) $\left(t=t^{n+1}\right)$ respectively from (14a) and (14b), we have

$$
\begin{aligned}
& \left(D_{x} \sigma^{x, n+1}+D_{y} \sigma^{y, n+1}+D_{z} \sigma^{z, n+1}, v\right)_{m}=0, \forall v \in S_{h}, \\
& \left(k^{-1}\left(\bar{C}^{x, n}\right) \sigma^{x, n+1}, w^{x}\right)_{x}+\left(k^{-1}\left(\bar{C}^{y, n}\right) \sigma^{y, n+1}, w^{y}\right)_{y}+\left(k^{-1}\left(\bar{C}^{z, n}\right) \sigma^{z, n+1}, w^{z}\right)_{z} \\
& -\left(\pi^{n+1}, D_{x} w^{x}+D_{y} w^{y}+D_{z} w^{z}\right)_{m}=-\left\{\left(\left(k^{-1}\left(\bar{C}^{x, n}\right)-k^{-1}\left(c^{n+1}\right)\right) \tilde{U}^{x, n+1}, w^{x}\right)_{x}\right. \\
& \left.+\left(\left(k^{-1}\left(\bar{C}^{y, n}\right)-k^{-1}\left(c^{n+1}\right)\right) \tilde{U}^{y, n+1}, w^{y}\right)_{y}+\left(\left(k^{-1}\left(\bar{C}^{z, n}\right)-k^{-1}\left(c^{n+1}\right)\right) \tilde{U}^{z, n+1}, w^{z}\right)_{z}\right\}, \forall \mathbf{w} \in V_{h} .
\end{aligned}
$$

Take $v=\pi^{n+1}$ in (29a), $\mathbf{w}=\sigma^{n+1}$ in (29b), and combine them together, we have

$$
\begin{aligned}
& \left(k^{-1}\left(\bar{C}^{x, n}\right) \sigma^{x, n+1}, \sigma^{x, n+1}\right)_{x}+\left(k^{-1}\left(\bar{C}^{y, n}\right) \sigma^{y, n+1}, \sigma^{y, n+1}\right)_{y}+\left(k^{-1}\left(\bar{C}^{z, n}\right) \sigma^{z, n+1}, \sigma^{z, n+1}\right)_{z} \\
& =-\sum_{s=x, y, z}\left(\left(k^{-1}\left(\bar{C}^{s, n}\right)-k^{-1}\left(c^{n+1}\right)\right) \tilde{U}^{s, n+1}, \sigma^{s, n+1}\right)_{s} .
\end{aligned}
$$

Eq.(30) is estimated by using Lemma 1-Lemma 5, the Taylor expansion and (C),

$$
\begin{aligned}
\left\|\sigma^{n+1}\right\|^{2} & \leq K \sum_{s=x, y, z}\left\|\bar{C}^{s, n}-c^{n+1}\right\|_{m}^{2} \leq K\left\{\sum_{s=x, y, z}\left\|\tilde{C}^{s, n}-c^{n}\right\|_{m}^{2}+\left\|\xi^{n}\right\|_{m}^{2}+\left\|\zeta^{n}\right\|_{m}^{2}+(\Delta t)^{2}\right\} \\
& \leq K\left\{\left\|\xi^{n}\right\|^{2}+h_{c}^{4}+(\Delta t)^{2}\right\} .
\end{aligned}
$$

$\pi^{n+1} \in S_{h}$ is discussed by using the method of duality (Jiang Pang, 1979; Nithsche, 1968) later. Consider the following elliptic problem

$$
\begin{aligned}
& \nabla \cdot \omega=\pi^{n+1}, X=(x, y, z)^{T} \in \Omega, \\
& \omega=\nabla p, X \in \Omega, \\
& \omega \cdot \gamma=0, X \in \partial \Omega .
\end{aligned}
$$

Using the regularity (32),

$$
\sum_{s=x, y, z}\left\|\frac{\partial \omega^{s}}{\partial s}\right\|_{m}^{2} \leq K\left\|\pi^{n+1}\right\|_{m}^{2} .
$$

If $\tilde{\omega} \in V_{h}$ satisfies

$$
\left(\frac{\partial \tilde{\omega}^{s}}{\partial s}, v\right)_{m}=\left(\frac{\partial \omega^{s}}{\partial s}, v\right)_{m}, \forall v \in S_{h}, s=x, y, z,
$$

then the solution $\tilde{\omega}$ exists and is estimated by

$$
\sum_{s=x, y, z}\left\|\frac{\partial \tilde{\omega}^{s}}{\partial s}\right\|_{m}^{2} \leq \sum_{s=x, y, z}\left\|\frac{\partial \omega^{s}}{\partial s}\right\|_{m}^{2} .
$$


Using Lemma 4, (32), (33) and (29), we have

$$
\begin{aligned}
& \left\|\pi^{n+1}\right\|_{m}^{2}=\left(\pi^{n+1}, \nabla \cdot \omega\right)=\left(\pi^{n+1}, D_{x} \tilde{\omega}^{x}+D_{y} \tilde{\omega}^{y}+D_{z} \tilde{\omega}^{z}\right)_{m} \\
& =\sum_{s=x, y, z}\left(k^{-1}\left(\bar{C}^{s, n}\right) \sigma^{s, n+1}, \tilde{\omega}^{s}\right)_{s}+\sum_{s=x, y, z}\left(\left(k^{-1}\left(\bar{C}^{s, n}\right)-k^{-1}\left(c^{n+1}\right)\right) \tilde{U}^{s, n+1}, \tilde{\omega}^{s}\right)_{s} \\
& \leq K\|\tilde{\omega}\|\left\{\left\|\left.\right|_{\sigma^{n+1}}\right\|^{2}+\left\|\xi^{n}\right\|_{m}^{2}+h_{c}^{4}+(\Delta t)^{2}\right\}^{1 / 2} .
\end{aligned}
$$

From Lemma 4, (33) and (34), we have

$$
\|\tilde{\omega}\|^{2} \leq \sum_{s=x, y, z}\left\|D_{s} \tilde{\omega}^{s}\right\|_{m}^{2}=\sum_{s=x, y, z}\left\|\frac{\partial \tilde{\omega}^{s}}{\partial s}\right\|_{m}^{2} \leq \sum_{s=x, y, z}\left\|\frac{\partial \omega^{s}}{\partial s}\right\|_{m}^{2} \leq K\left\|\pi^{n+1}\right\|_{m}^{2} .
$$

Substituting (36) into (35),

$$
\left\|\pi^{n+1}\right\|_{m}^{2} \leq K\left\{\left\|\sigma^{n+1} \mid\right\|^{2}+\left\|\xi^{n}\right\|_{m}^{2}+h_{c}^{4}+(\Delta t)^{2}\right\} \leq K\left\{\left\|\xi^{n}\right\|_{m}^{2}+h_{c}^{4}+(\Delta t)^{2}\right\} .
$$

Error function $\mu$ is discussed later. It follows from (3) $\left(t=t^{n+1}\right),(16)$ and (26),

$$
\begin{aligned}
\left(\frac{\mu^{n+1}-\mu^{n}}{\Delta t}, \varphi\right) & =\hat{\alpha}\left(\phi\left(C^{n}\right)-\phi\left(\tilde{C}^{n}\right)+\phi\left(\tilde{C}^{n}\right)-\phi\left(C^{n+1}\right), \varphi\right)-\hat{\alpha}\left(\theta^{n}, \varphi\right)-\hat{\alpha}\left(S^{n+1}-S^{n}, \varphi\right) \\
& -\left(\frac{\partial \tilde{s}^{n+1}}{\partial t}-\frac{s^{n+1}-s^{n}}{\Delta t}, \varphi\right), \forall \varphi \in M_{h} .
\end{aligned}
$$

For the Langmuir isotherm, it is easy to see that $\phi^{\prime}(c) \leq k_{1}$ holds from $\phi(c)=\frac{k_{1} c}{1+k_{2} c}$ and $\phi^{\prime}(c)=\frac{k_{1}}{\left(1+k_{2} c\right)^{2}}$. For the Freundlinch isotherm, $\phi(c)=k_{3} c^{p}, \phi^{\prime}(c)=k_{3} p c^{p-1}$, let $p=1$, then $f^{\prime}(c) \leq k_{3}$. The analysis proceeds by applying the Lagrange mean value theorem. Taking $\varphi=\partial_{t} \mu^{n}=\frac{\mu^{n+1}-\mu^{n}}{\Delta t}$ in (38), using Lemma 5, Lemma 6 and the regularity (R), we have

$$
\left\|\partial_{t} \mu^{n}\right\|_{L^{2}}^{2} \leq K\left\{\left\|\xi^{n}\right\|_{L^{2}}^{2}+\left\|\mu^{n}\right\|_{L^{2}}^{2}+h_{c}^{4}+h_{p}^{4}+(\Delta t)^{2}\right\}
$$

Taking $\varphi=\mu^{n+1}$ in (38), we have

$$
\frac{1}{2 \Delta t}\left\{\left\|\mu^{n+1}\right\|_{L^{2}}^{2}-\left\|\mu^{n}\right\|_{L^{2}}^{2}\right\} \leq K\left\{\left\|\mu^{n+1}\right\|_{L^{2}}^{2}+\left\|\mu^{n}\right\|_{L^{2}}^{2}+\left\|\xi^{n}\right\|_{L^{2}}^{2}+h_{c}^{4}+h_{p}^{4}+(\Delta t)^{2}\right\} .
$$

Next, eq.(2) is estimated. Subtracting (25a) and (25b) $t=t^{n+1}$ respectively from (15a) and (15b), and taking $v=\xi^{n+1}$ and $w=\alpha^{n+1}$, we have

$$
\begin{aligned}
& \left(\theta \frac{C^{n+1}-\hat{C}^{n}}{\Delta t}, \xi^{n+1}\right)_{m}+\left(\rho \frac{C^{\prime n+1}-C^{\prime n}}{\Delta t}, \xi^{n+1}\right)_{m}+\left(D_{x} \alpha^{x, n+1}+D_{y} \alpha^{y, n+1}+D_{z} \alpha^{z, n+1}, \xi^{n+1}\right)_{m} \\
& =\left(f\left(\hat{C}^{n}\right)-f\left(c^{n+1}\right)+\psi^{n+1} \frac{\partial c^{n+1}}{\partial \tau}+\rho \frac{\partial c^{\prime n+1}}{\partial t}, \xi^{n+1}\right)_{m}, \\
& \left.\left(D^{-1} \alpha^{x, n+1}, \alpha^{x, n+1}\right)_{x}+\left(D^{-1} \alpha^{y, n+1}, \alpha^{y, n+1}\right)_{y}+\left(D^{-1} \alpha^{z, n+1}\right), \alpha^{z, n+1}\right)_{z} \\
& -\left(\xi^{n+1}, D_{x} \alpha^{x, n+1}+D_{y} \alpha^{y, n+1}+D_{z} \alpha^{z, n+1}\right)_{m}=0 .
\end{aligned}
$$

Considering the sum of (40a) and (40b),

$$
\begin{aligned}
& \left(\theta \frac{C^{n+1}-\hat{C}^{n}}{\Delta t}, \xi^{n+1}\right)_{m}+\left(\rho \frac{C^{\prime n+1}-C^{\prime n}}{\Delta t}, \xi^{n+1}\right)_{m}+\left(D^{-1} \alpha^{x, n+1}, \alpha^{x, n+1}\right)_{x} \\
& +\left(D^{-1} \alpha^{y, n+1}, \alpha^{y, n+1}\right)_{y}+\left(D^{-1} \alpha^{z, n+1}, \alpha^{z, n+1}\right)_{z} \\
& =\left(f\left(\hat{C}^{n}\right)-f\left(c^{n+1}\right)+\psi^{n+1} \frac{\partial c^{n+1}}{\partial \tau}+\rho \frac{\partial c^{\prime n+1}}{\partial t}, \xi^{n+1}\right)_{m} .
\end{aligned}
$$


Subtracting (2) $t=t^{n+1}$ from (41), we get the error equation

$$
\begin{aligned}
& \left(\theta \frac{\xi^{n+1}-\xi^{n}}{\Delta t}, \xi^{n+1}\right)_{m}+\sum_{s=x, y, z}\left(D^{-1} \alpha^{s, n+1}, \alpha^{s, n+1}\right)_{s} \\
& =-\left(\rho \frac{\mu^{n+1}-\mu^{n}}{\Delta t}, \xi^{n+1}\right)_{m}+\left(\rho\left(\frac{\partial s^{n+1}}{\partial t}-\frac{s^{n+1}-s^{n}}{\Delta t}\right), \xi^{n+1}\right)_{m}-\left(\rho \partial_{t} \lambda^{n}, \xi^{n}\right) \\
& +\left(\left[\theta \frac{\partial c^{n+1}}{\partial t}+\mathbf{u}^{n+1} \cdot \nabla c^{n+1}\right]-\theta \frac{c^{n+1}-\check{c}^{n}}{\Delta t}, \xi^{n+1}\right)_{m}+\left(\theta \frac{\zeta^{n+1}-\zeta^{n}}{\Delta t}, \xi^{n+1}\right)_{m} \\
& +\left(f\left(\hat{C}^{n}\right)-f\left(c^{n+1}\right), \xi^{n+1}\right)+\left(\theta \frac{\hat{c}^{n}-\check{c}^{n}}{\Delta t}, \xi^{n+1}\right)_{m}-\left(\theta \frac{\hat{\zeta}^{n}-\check{\zeta}^{n}}{\Delta t}, \xi^{n+1}\right)_{m} \\
& +\left(\theta \frac{\hat{\xi}^{n}-\check{\xi}^{n}}{\Delta t}, \xi^{n+1}\right)_{m}-\left(\theta \frac{\check{\zeta}^{n}-\zeta^{n}}{\Delta t}, \xi^{n+1}\right)_{m}+\left(\theta \frac{\check{\xi}^{n}-\xi^{n}}{\Delta t}, \xi^{n+1}\right)_{m},
\end{aligned}
$$

where $\check{c}^{n}=c^{n}\left(X-\theta^{-1} \mathbf{u}^{n+1} \Delta t\right), \hat{c}^{n}=c^{n}\left(X-\theta^{-1} \mathbf{U}^{n+1} \Delta t\right), \cdots$.

The inequality $a(a-b) \geq \frac{1}{2}\left(a^{2}-b^{2}\right)$ is used to estimate the left-hand term of (42). The terms on the right-hand side are denoted by $T_{1}, T_{2}, \cdots, T_{11}$. Then,

$$
\frac{1}{2 \Delta t}\left\{\left(\theta \xi^{n+1}, \xi^{n+1}\right)_{m}-\left(\theta \xi^{n}, \xi^{n}\right)_{m}\right\}+\sum_{s=x, y, z}\left(D^{-1} \alpha^{s, n+1}, \alpha^{s, n+1}\right)_{s} \leq \sum_{i=1}^{11} T_{i} .
$$

$T_{1}, T_{2}$ and $T_{3}$ of (43) are estimated by using the relation of continuous $L^{2}$-norm and discrete $m$-norm (Douglas, 1982; Raviart \& Thomas, 1977) and (39),

$$
\begin{aligned}
\left|T_{1}+T_{2}+T_{3}\right| & \leq K\left\|\partial_{t} \mu^{n}\right\|_{m}^{2}+K\left\{\left\|\xi^{n+1}\right\|_{m}^{2}+h_{c}^{4}+(\Delta t)^{2}\right\} \\
& \leq K\left\{\left\|\xi^{n+1}\right\|_{m}^{2}+\left\|\xi^{n}\right\|_{m}^{2}+\left\|\mu^{n}\right\|_{m}^{2}+h_{c}^{4}+h_{p}^{4}+(\Delta t)^{2}\right\} .
\end{aligned}
$$

Noting that $\theta \frac{\partial c^{n+1}}{\partial t}+\mathbf{u}^{n+1} \cdot \nabla c^{n+1}=\psi^{n+1} \frac{\partial c^{n+1}}{\partial \tau}$, we have

$$
\frac{\partial c^{n+1}}{\partial \tau}-\frac{\theta}{\psi^{n+1}} \frac{c^{n+1}-\check{c}^{n}}{\Delta t}=\frac{\theta}{\psi^{n+1} \Delta t} \int_{\left(\check{X}, t^{n}\right)}^{\left(X, t^{n+1}\right)}\left[|X-\hat{X}|^{2}+\left(t-t^{n}\right)^{2}\right]^{1 / 2} \frac{\partial^{2} c}{\partial \tau^{2}} d \tau .
$$

Multiplying both sides by $\psi^{n+1}$ and estimating it in $m$-norm, we have

$$
\begin{aligned}
& \left\|\psi^{n+1} \frac{\partial c^{n+1}}{\partial \tau}-\theta \frac{c^{n+1}-\check{c}^{n}}{\Delta t}\right\|_{m}^{2} \leq \int_{\Omega}\left[\frac{\psi^{n+1}}{\Delta t}\right]^{2}\left|\int_{\left(\check{X}, t^{n}\right)}^{\left(X, t^{n+1}\right)} \frac{\partial^{2} c}{\partial \tau^{2}} d \tau\right|^{2} d X \\
& \leq \Delta t\left\|\frac{\left(\psi^{n+1}\right)^{3}}{\theta}\right\|_{\infty} \int_{\Omega} \int_{\left(\check{X}, t^{n}\right)}^{\left(X, t^{n+1}\right)}\left|\frac{\partial^{2} c}{\partial \tau^{2}}\right|^{2} d \tau d X \\
& \leq \Delta t\left\|\frac{\left(\psi^{n+1}\right)^{4}}{\theta^{2}}\right\|_{\infty} \int_{\Omega} \int_{t^{n}}^{t^{n+1}} \int_{0}^{1}\left|\frac{\partial^{2} c}{\partial \tau^{2}}(\bar{\tau} \check{X}+(1-\bar{\tau}) X, t)\right|^{2} d \bar{\tau} d X d t .
\end{aligned}
$$

Therefore, $T_{4}$ is bounded by

$$
\left|T_{4}\right| \leq K\left\|\frac{\partial^{2} c}{\partial \tau^{2}}\right\|_{L^{2}\left(t^{n}, t^{n+1} ; m\right)}^{2} \Delta t+K\left\|\xi^{n+1}\right\|_{m}^{2} .
$$

From Lemma 5, $T_{5}$ and $T_{6}$ are estimated similarly

$$
\begin{gathered}
\left|T_{5}\right| \leq K\left\{(\Delta t)^{-1}\left\|\frac{\partial \zeta}{\partial t}\right\|_{L^{2}\left(t^{n}, t^{n+1} ; m\right)}^{2}+\left\|\xi^{n+1}\right\|_{m}^{2}\right\} \\
\left|T_{6}\right| \leq K\left\{\left\|\xi^{n+1}\right\|_{m}^{2}+\left\|\xi^{n}\right\|_{m}^{2}+(\Delta t)^{2}+h^{4}\right\} .
\end{gathered}
$$


$T_{7}, T_{8}$ and $T_{9}$ are argued as follows. If $f$ is a function defined on $\Omega$, denoting one of three functions $c, \zeta$ and $\xi$, and $Z$ means the unit vector of $\mathbf{U}^{n+1}-\mathbf{u}^{n+1}$, then

$$
\begin{aligned}
& \int_{\Omega} \theta \frac{\hat{f}^{n}-\check{f}^{n}}{\Delta t} \xi^{n+1} d X=(\Delta t)^{-1} \int_{\Omega} \theta\left[\int_{\check{X}}^{\hat{X}} \frac{\partial f^{n}}{\partial Z} d Z\right] \xi^{n+1} d X \\
& =(\Delta t)^{-1} \int_{\Omega} \theta\left[\int_{0}^{1} \frac{\partial f^{n}}{\partial Z}((1-\bar{Z}) \check{X}+\bar{Z} \hat{X}) d \bar{Z}\right]|\hat{X}-\check{X}| \xi^{n+1} d X \\
& =\int_{\Omega}\left[\int_{0}^{1} \frac{\partial f^{n}}{\partial Z}((1-\bar{Z}) \check{X}+\bar{Z} \hat{X}) d \bar{Z}\right]|\mathbf{u}-\mathbf{U}| \xi^{n+1} d X,
\end{aligned}
$$

where the parameter $\bar{Z} \in[0,1], \hat{X}-\check{X}=\Delta t\left[\mathbf{u}^{n+1}(X)-\mathbf{U}^{n+1}(X)\right] / \theta(X)$. Define

$$
g_{f}=\int_{0}^{1} \frac{\partial f^{n}}{\partial Z}((1-\bar{Z}) \check{X}+\bar{Z} \hat{X}) d \bar{Z},
$$

then we obtain three special inequalities from (48),

$$
\begin{aligned}
& \left|T_{7}\right| \leq\left\|g_{c}\right\|_{\infty}\left\|(\mathbf{u}-\mathbf{U})^{n+1}\right\|_{m}\left\|\xi^{n+1}\right\|_{m}, \\
& \left|T_{8}\right| \leq\left\|g_{\xi}\right\|_{m}\left\|(\mathbf{u}-\mathbf{U})^{n+1}\right\|_{m}\left\|\xi^{n+1}\right\|_{\infty}, \\
& \left|T_{9}\right| \leq\left\|g_{\xi}\right\|_{m}\left\|(\mathbf{u}-\mathbf{U})^{n+1}\right\|_{m}\left\|\xi^{n+1}\right\|_{\infty} .
\end{aligned}
$$

From Lemma 1-Lemma 5, and (31), we have

$$
\left\|(\mathbf{u}-\mathbf{U})^{n+1}\right\|_{m}^{2} \leq K\left\{\left\|\xi^{n}\right\|_{m}^{2}+h_{p}^{4}+h_{c}^{4}+(\Delta t)^{2}\right\} .
$$

Since $g_{c}(X)$ is the mean value of first-order partial derivatives of $c^{n}$, it could be estimated by $\left\|c^{n}\right\|_{W_{\infty}^{1}}$. It follows from (49a),

$$
\left|T_{7}\right| \leq K\left\{\left\|\xi^{n+1}\right\|_{m}^{2}+\left\|\xi^{n}\right\|_{m}^{2}+h_{p}^{4}+h_{c}^{4}+(\Delta t)^{2}\right\} .
$$

In order to estimate $\left\|g_{\zeta}\right\|_{m}$ and $\left\|g_{\xi}\right\|_{m}$, we introduce the following induction hypothesis

$$
\sup _{0 \leq n \leq L}\|\sigma \mid\|_{\infty} \rightarrow 0, \sup _{0 \leq n \leq L}\left\|\xi^{n}\right\|_{m} \rightarrow 0,\left(h_{c}, h_{p}, \Delta t\right) \rightarrow 0,
$$

and the partition restriction

$$
\Delta t=O\left(h^{2}\right), h^{2}=o\left(h_{p}^{3 / 2}\right) .
$$

Considering

$$
\left\|g_{f}\right\|^{2} \leq \int_{0}^{1} \int_{\Omega}\left[\frac{\partial f^{n}}{\partial Z}((1-\bar{Z}) \check{X}+\bar{Z} \hat{X})\right]^{2} d X d \bar{Z} .
$$

Using the transformation,

$$
G_{\bar{Z}}(X)=(1-\bar{Z}) \check{X}+\bar{Z} \hat{X}=X-\left[\theta^{-1}(X) \mathbf{u}^{n+1}(X)+\bar{Z} \theta^{-1}(X)(\mathbf{U}-\mathbf{u})^{n+1}(X)\right] \Delta t,
$$

then, we have

$$
\left\|g_{f}\right\|^{2} \leq \int_{0}^{1} \sum_{J_{p}}\left|\frac{\partial f^{n}}{\partial Z}\left(G_{\bar{Z}}(X)\right)\right|^{2} d X d \bar{Z},
$$

where $J_{p}=\Omega_{i j k}=\left[x_{i-1 / 2}, x_{i+1 / 2}\right] \times\left[y_{j-1 / 2}, y_{j+1 / 2}\right] \times\left[z_{k-1 / 2}, z_{k+1 / 2}\right]$ denotes a grid element of the flow equation.

It follows from (52) and (53),

$$
\operatorname{det} G_{\bar{Z}}=1+o(1)
$$

Then, eq.(56) is estimated by

$$
\left\|g_{f}\right\|^{2} \leq K\left\|\nabla f^{n}\right\|^{2} .
$$

Using (57), Lemma 5 and the Sobolev embedding theorem (Adams, 1975), we could estimate $T_{8}$,

$$
\begin{aligned}
\left|T_{8}\right| & \leq K\left\|\nabla \zeta^{n}\right\| \cdot\left\|(\mathbf{u}-\mathbf{U})^{n+1}\right\| \cdot h^{-(\varepsilon+1 / 2)}\left\|\nabla \xi^{n+1}\right\| \\
& \leq K\left\{h_{c}^{2-(\varepsilon+1 / 2)}\left\|(\mathbf{u}-\mathbf{U})^{n+1}\right\|\left\|\nabla \xi^{n+1}\right\|\right\} \\
& \leq K\left\{\left\|\xi^{n+1}\right\|_{m}^{2}+\left\|\xi^{n}\right\|_{m}^{2}+h_{p}^{4}+h_{c}^{4}+(\Delta t)^{2}\right\}+\varepsilon\left\|\alpha^{n+1}\right\|^{2} .
\end{aligned}
$$


From (50), we find that $\left\|(\mathbf{u}-\mathbf{U})^{n+1}\right\|_{m}=o\left(h_{c}^{-(\varepsilon+1 / 2)}\right)$. Therefore, it is necessary to prove $\left\|\xi^{n}\right\|_{m}=O\left(h_{p}^{2}+h_{c}^{2}+\Delta t\right)$. Using the argument similar to the reference (Yuan, 2013), we have

$$
\begin{aligned}
\left|T_{9}\right| & \leq K\left\|\nabla \xi^{n}\right\| \cdot\left\|(\mathbf{u}-\mathbf{U})^{n+1}\right\| \cdot h^{-(\varepsilon+1 / 2)}\left\|\nabla \xi^{n+1}\right\| \\
& \leq \varepsilon\left\{\left\|\mid \alpha^{n+1}\right\|\left\|^{2}+\right\| \alpha^{n} \|^{2}\right\} .
\end{aligned}
$$

$T_{10}$ and $T_{11}$ are treated by using the negative norm estimates

$$
\begin{aligned}
& \left|T_{10}\right| \leq K h_{c}^{4}+\varepsilon\left|\left\|\alpha^{n+1}\right\|\right|^{2}, \\
& \left|T_{11}\right| \leq K\left\|\xi^{n}\right\|_{m}^{2}+\varepsilon\left\|\left|\alpha^{n+1}\right|\right\|^{2} .
\end{aligned}
$$

Considering the resulting estimates (43), (44), (47), (51), (58), (59) and (42) together, we have

$$
\begin{aligned}
& \frac{1}{2 \Delta t}\left\{\left(\theta \xi^{n+1}, \xi^{n+1}\right)_{m}-\left(\theta \xi^{n}, \xi^{n}\right)_{m}\right\}+\sum_{s=x, y, z}\left(D^{-1} \alpha^{s, n+1}, \alpha^{s, n+1}\right)_{s} \\
& \leq K\left\{\left\|\frac{\partial^{2} c}{\partial \tau^{2}}\right\|_{L^{2}\left(t^{n}, t^{n+1} ; m\right)}^{2} \Delta t+(\Delta t)^{-1}\left\|\frac{\partial \zeta}{\partial t}\right\|_{L^{2}\left(t^{n}, t^{n+1} ; m\right)}^{2}+\left\|\xi^{n+1}\right\|_{m}^{2}+\left\|\xi^{n}\right\|_{m}^{2}+\left\|\mu^{n}\right\|_{m}^{2}\right. \\
& \left.\quad+h_{p}^{4}+h_{c}^{4}+(\Delta t)^{2}\right\}+\varepsilon\left\{\left.\left\|\alpha^{n+1}\right\|\right|^{2}+\left\|\alpha^{n}\right\|^{2}\right\} .
\end{aligned}
$$

Multiplying (60) by $2 \Delta t$, summing them on $n(0 \leq n \leq L)$, and noting that $\xi^{0}=0$, we have

$$
\left\|\xi^{L+1}\right\|_{m}^{2}+\sum_{n=0}^{L}\left\|\alpha^{n+1}\right\| \|^{2} \Delta t \leq K\left\{\sum_{n=0}^{L}\left[\left\|\xi^{n+1}\right\|_{m}^{2}+\left\|\mu^{n}\right\|_{m}^{2}\right] \Delta t+h_{p}^{4}+h_{c}^{4}+(\Delta t)^{2}\right\} .
$$

It holds for (39b) from $\mu^{0}=0$,

$$
\left\|\mu^{L+1}\right\|_{L^{2}}^{2} \leq K\left\{\sum_{n=1}^{L}\left[\left\|\mu^{n}\right\|_{L^{2}}^{2}+\left\|\xi^{n}\right\|_{L^{2}}^{2}\right] \Delta t+h_{c}^{4}+h_{p}^{4}+(\Delta t)^{2}\right\} .
$$

Considering (61), (62) and the relation of continuous $L^{2}$-norm and discrete $m$-norm (Douglas, 1982; Raviart \& Thomas, 1977) together, we obtain

$$
\left\|\xi^{L+1}\right\|_{m}^{2}+\left\|\mu^{L+1}\right\|_{L^{2}}^{2}+\sum_{n=0}^{L}\left\|\alpha^{n+1}\right\|^{2} \Delta t \leq K\left\{\sum_{n=0}^{L}\left[\left\|\xi^{n+1}\right\|_{m}^{2}+\left\|\mu^{n}\right\|_{L^{2}}^{2}\right] \Delta t+h_{p}^{4}+h_{c}^{4}+(\Delta t)^{2}\right\} .
$$

Applying the Gronwall Lemma, we get

$$
\left\|\xi^{L+1}\right\|_{m}^{2}+\left\|\mu^{L+1}\right\|_{L^{2}}^{2}+\sum_{n=0}^{L}\left\|\alpha^{n+1}\right\|^{2} \Delta t \leq K\left\{h_{p}^{4}+h_{c}^{4}+(\Delta t)^{2}\right\} .
$$

By (64a), (31) and (37), we have

$$
\sup _{0 \leq n \leq L}\left\{\left\|\pi^{n+1}\right\|_{m}^{2}+\left\|\alpha^{n+1}\right\|^{2}\right\} \leq K\left\{h_{p}^{4}+h_{c}^{4}+(\Delta t)^{2}\right\} .
$$

It remains to testify the induction hypothesis (52). For $n=0$, it is true obviously by using the initial conditions and $\xi^{0}=0$. Suppose that it holds for $1 \leq n \leq L$. Then, it follows from (64) and (53)

$$
\begin{aligned}
& \left\|\sigma^{L+1}\right\|_{\infty} \leq K h_{p}^{-3 / 2}\left\{h_{p}^{2}+h_{c}^{2}+\Delta t\right\} \leq K h_{p}^{1 / 2} \rightarrow 0, \\
& \left\|\xi^{L+1}\right\|_{\infty} \leq K h_{c}^{-3 / 2}\left\{h_{p}^{2}+h_{c}^{2}+\Delta t\right\} \leq K h_{c}^{1 / 2} \rightarrow 0 .
\end{aligned}
$$

Therefore, it holds for $n=L+1$ and the proof is completed.

The following theorem is concluded from (64), Lemma 5 and Lemma 6. 
Theorem 3. Suppose that the conditions (R) and (C) hold. Numerical solutions are obtained by using the procedures (14), (15) and (16). If the restriction (53) holds, then

$$
\begin{aligned}
& \|p-P\|_{\bar{L}^{\infty}(J ; m)}+\|\mathbf{u}-\mathbf{U}\|_{\bar{L}^{\infty}(J ; V)}+\|c-C\|_{\bar{L}^{\infty}(J ; m)}+\|\mathbf{g}-\mathbf{G}\|_{\bar{L}^{2}(J ; V)}+\|s-S\|_{\bar{L}^{\infty}\left(J ; L^{2}\right)} \\
& \leq M^{*}\left\{h_{p}^{2}+h_{c}^{2}+\Delta t\right\},
\end{aligned}
$$

where $\|g\|_{\bar{L}^{\infty}(J ; X)}=\sup _{n \Delta t \leq T}\left\|g^{n}\right\|_{X},\|g\|_{\bar{L}^{2}(J ; X)}=\sup _{L \Delta t \leq T}\left\{\sum_{n=0}^{L}\left\|g^{n}\right\|_{X}^{2} \Delta t\right\}^{1 / 2}$, and the constant $M^{*}$ depends on $p, c, s$ and their derivatives.

\section{Modified Mixed Volume Element With Characteristic Volume Element and Its Numerical Analysis}

In $\$ 3$ and $\$ 4$, we discuss the method of mixed volume element with characteristic volume element. While in actual applications, Darcy velocity changes more slow than the concentration with respect to $t$. Therefore, a large time step may be used for solving eq.(1). The interval $J$ is partitioned by $0=t_{0}<t_{1}<\cdots<t_{L}=T$. Let $\Delta t_{p}^{m}=t_{m}-t_{m-1}$, and suppose that the partition is measured uniformly by $\Delta t_{p}^{m}=\Delta t_{p}, m \geq 2$ except $\Delta t_{p}^{1}$. The concentration is computed by a small uniform step $\Delta t_{c}=t^{n}-t^{n-1}$. Suppose that there exists a positive integer $n$ corresponding to $m$ such that the pressure and the concentration have the same nodes, $t_{m}=t^{n}$. Let $j=\Delta t_{p} / \Delta t_{c}$ and $j_{1}=\Delta t_{p}^{1} / \Delta t_{c}$. Let $\psi_{m}(X)=\psi\left(X, t_{m}\right)$. Darcy velocity $\mathbf{u}^{n+1}$ at $t^{n+1}, t_{m-1}<t^{n+1} \leq t_{m}$, in (15) is defined as follows. The approximation is defined by a linear extrapolation of $\mathbf{U}_{m-1}$ and $\mathbf{U}_{m-2}$ for $m \geq 2$

$$
E \mathbf{U}^{n+1}=\left(1+\frac{t^{n+1}-t_{m-1}}{t_{m-1}-t_{m-2}}\right) \mathbf{U}_{m-1}-\frac{t^{n+1}-t_{m-1}}{t_{m-1}-t_{m-2}} \mathbf{U}_{m-2}
$$

If $m=1$, define $E \mathbf{U}^{n+1}=\mathbf{U}_{0}$.

The procedures of (1) and (2) are formulated. Numerical solutions $\left(\mathbf{U}_{m}, P_{m}\right):\left(t_{0}, t_{1}, \cdots, t_{L}\right) \rightarrow S_{h} \times V_{h}$ and $\left(C^{n}, \mathbf{G}^{n}, C^{\prime n}\right):$ $\left(t^{0}, t^{1}, \cdots, t^{R}\right) \rightarrow S_{h} \times V_{h} \times M_{h}$ are computed by

$$
\begin{aligned}
& \left(D_{x} U_{m}^{x}+D_{y} U_{m}^{y}+D_{z} U_{m}^{z}, v\right)_{\hat{m}}=\left(q_{m}, v\right)_{\hat{m}}, \forall v \in S_{h}, \\
& \left(k^{-1}\left(\bar{C}_{m}^{x}\right) U_{m}^{x}, w^{x}\right)_{x}+\left(k^{-1}\left(\bar{C}_{m}^{y}\right) U_{m}^{y}, w^{y}\right)_{y}+\left(k^{-1}\left(\bar{C}_{m}^{z}\right) U_{m}^{z}, w^{z}\right)_{z} \\
& -\left(P_{m}, D_{x} w^{x}+D_{y} w^{y}+D_{z} w^{z}\right)_{\hat{m}}=0, \forall \mathbf{w} \in V_{h},
\end{aligned}
$$

For clarity, the previous $m$-norm is replaced by $\hat{m}$-norm.

$$
\begin{aligned}
& \left(\theta \frac{C^{n+1}-\hat{C}^{n}}{\Delta t}, v\right)_{\hat{m}}+\left(\rho \frac{C^{\prime n+1}-C^{\prime n}}{\Delta t}, v\right)_{\hat{m}}+\left(D_{x} G^{x, n+1}+D_{y} G^{y, n+1}+D_{z} G^{z, n+1}, v\right)_{\hat{m}} \\
& =\left(f\left(\hat{C}^{n}\right), v\right)_{\hat{m}}, \forall v \in S_{h}, \\
& \left(D^{-1} G^{x, n+1}, w^{x}\right)_{x}+\left(D^{-1} G^{y, n+1}, w^{y}\right)_{y}+\left(D^{-1} G^{z, n+1}, w^{z}\right)_{z}-\left(C^{n+1}, D_{x} w^{x}+D_{y} w^{y}+D_{z} w^{z}\right)_{\hat{m}} \\
& =0, \forall \mathbf{w} \in V_{h} .
\end{aligned}
$$

$$
\left(\frac{S^{n+1}-S^{n}}{\Delta t}, \varphi\right)_{L^{2}}=\hat{\alpha}\left(\phi\left(C^{n}\right)-S^{n}, \varphi\right)_{L^{2}}, \quad \forall \varphi \in M_{h}
$$

where $\hat{C}^{n}=C^{n}\left(X-\theta^{-1} E \mathbf{U}^{n+1} \Delta t\right)$.

$$
C^{0}=\tilde{C}^{0}, \quad G^{0}=\tilde{G}^{0}, \quad S^{0}=\tilde{S}^{0}, X \in \Omega .
$$

The procedures (68), (69), (70) and (71) are carried out similarly to (14)-(17) of $§ 3$. Here it is pointed out that eqs.(1) and (2) are discretized with two different steps. It can shorten the computational work greatly without losing accuracy.

In a similar analysis to Theorem 3, we conclude the following result.

Theorem 4. Suppose that exact solutions of (1)-(7) are regular (R), and the coefficients are positive definite (C). Adopting the procedures (68)-(71) to obtain numerical solutions, under the restriction (53), we have

$$
\begin{aligned}
& \|p-P\|_{L^{\infty}(J ; m)}+\|\mathbf{u}-\mathbf{U}\|_{\bar{L}^{\infty}(J ; V)}+\|c-C\|_{\bar{L}^{\infty}(J ; m)}+\|\mathbf{g}-\mathbf{G}\|_{\bar{L}^{2}(J ; V)}+\|s-S\|_{\bar{L}^{\infty}\left(J ; L^{2}\right)} \\
& \leq M^{* *}\left\{h_{p}^{2}+h_{c}^{2}+\Delta t_{c}+\left(\Delta t_{p}\right)^{2}+\left(\Delta t_{p}^{1}\right)^{3 / 2}\right\},
\end{aligned}
$$

where the constant $M^{* *}$ depends on $p, c, s$ and their derivatives. 


\section{Conclusions and Discussions}

Numerical approximation and theoretical analysis of three-dimensional contamination transport are considered in this paper. A type of mixed volume element-characteristic mixed volume element method is discussed. Several interesting conclusions are stated as follows.

(I) The method has the physical nature of conservation (local and whole), an important physical nature in numerical simulation of underground environmental science problems.

(II) The composite method combines a mixed volume element and the idea of characteristics, so it has strong stability and high accuracy. It is quite useful especially in large-scaled engineering computation on a three-dimensional complicated region.

(III) The method develops the convergence result of 3/2-order of Arbogast and Wheeler to second order, and possibly solves the problems better (Arbogast \& Wheeler, 1995; Ewing, 1983; Shen, Liu \& Tang, 2002; Vogt, 1982).

\section{Acknowledgements}

This work is supported by the National Natural Science Foundation of China (Grant No. 11871312).

\section{References}

Adams, R. A. (1975). Sobolev Spaces. Academic Press, New York.

Arbogast, T., \& Wheeler, M. F. (1995). A characteristics-mixed finite element methods for advection-dominated transport problems. SIAMJ. Numer. Anal., 32(2), 404-424. https://doi.org/10.1137/0732017

Bell, J. B., Dawson, C. N., \& Shubin, G. R. (1988). An unsplit high-order Godunov scheme for scalar conservation laws in two dimensions. J. Comput. Phys., 74, 1-24. https://doi.org/10.1016/0021-9991(88)90065-4

Cai, Z. (1991). On the finite volume element method. Numer. Math., 58, 713-735. https://doi.org/10.1007/BF01385651

Cai, Z., Jones, J. E., Mccormilk, S. F., \& Russell, T. F. (1997). Control-volume mixed finite element methods. Comput. Geosci., 1, 289-315.

Cella, M. A., Russell, T. F., Herrera, I., \& Ewing, R. E. (1990). An EulerianCLagrangian localized adjoint method for the advectionCdiffusion equations. Adv. Water Resour., 13(4), 187-206.

Cialet, P. G. (1978) The finite element method for elliptic problems. North Holland, Amsterdam.

Chou, S. H., Kawk, D. Y., \& Vassileviki, P. (1998). Mixed volume methods for elliptic problems on triangular grids. SIAM J. Numer. Anal., 35(5), 1850-1861. https://doi.org/10.1137/S0036142997321285

Chou, S. H., \& Vassileviki, P. (1999). A general mixed covolume framework for constructing conservative schemes for elliptic problems. Math. Comp., 68(227), 991-1011. https://doi.org/10.1090/S0025-5718-99-01090-X

Chou, S. H., Kawk, D. Y., \& Vassileviki, P. (2000). Mixed volume methods on rectangular grids for elliptic problem. SIAM J. Numer. Anal., 37(3), 758-771. https://doi.org/10.1137/S0036142996305534

Dawson, C. N., Russell, T. F., \& Wheeler, M. F. (1989). Some improved error estimates for the modified method of characteristics. SIAM J. Numer. Anal., 26(6), 1487-1512. https://doi.org/10.1137/0726087

Dawson, C. N., Van Duiji, C. J., \& Wheeler, M. F. (1994). Characteristic-Galerkin methods for contaminant transport with nonequilibrium adsorption kinetics. SIAM J. Numer. Anal., 31(4), 982-999. https://doi.org/10.1137/0731052

Dawson, C. N. (1998). Analysis of an upwind-mixed finite element method for nonlinear contaminant transport equations. SIAM J. Numer. Anal., 35(5), 1709-1724. https://doi.org/10.1137/S0036142993259421

Douglas Jr., J. (1982). Simulation of miscible displacement in porous media by a modified method of characteristic procedure. In Numerical Analysis, Dundee, 1981. Lecture Notes in Mathematics, 912, Springer-Verlag, Berlin. https://doi.org/10.1007/BFb0093149

Douglas Jr., J., Ewing, R. E., \& Wheeler, M. F. (1983). Approximation of the pressure by a mixed method in the simulation of miscible displacement. RAIRO Anal. Numer., 17(1), 17-33. https://doi.org/10.1051/m2an/1983170100171

Douglas Jr., J., Ewing, R. E., \& Wheeler, M. F. (1983). A time-discretization procedure for a mixed finite element approximation of miscible displacement in porous media. RAIRO Anal. Numer., 17(3), 249-265.

Ewing, R. E. (1983). The mathematics of reservoir simulation. SIAM, Philadelphia. https://doi.org/10.1137/1.9781611971071 
Hornung, U. (1988). Miscible displacement in porous media influenced by mobile and immobile water. Nonlinear Partial Differential Equation, Springer, New York.

Li, R. H., \& Chen, Z. Y. (1994). Generalized difference of differential equations. Jilin University Press, Changchun. (In Chinese)

Jiang, L. S., \& Pang, Z. Y. (1979). Finite element method and its theory. People's Education Press, Beijing. (In Chinese)

Johnson, C. (1986). Streamline diffusion methods for problems in fluid mechanics. in Finite Element in Fluids VI, Wiley, New York.

Jones, J. E. (1995). A mixed volume method for accurate computation of fluid velocities in porous media. $\mathrm{Ph}$. $\mathrm{D}$. Thesis. University of Colorado, Denver, Co.

Nitsche, J. (1968). Linear splint-funktionen and die methoden von Ritz for elliptishce randwert probleme. Arch. for Rational Mech. Anal., 36, 348-355.

Pan, H., \& Rui, H. X. (2012). Mixed element method for two-dimensional Darcy-Forchheimer model. J. Sci. Comput., 52(3), 563-587. https://doi.org/10.1007/s10915-011-9558-3

Raviart, P. A., \& Thomas, J. M. (1977). A mixed finite element method for second order elliptic problems, in: Mathematical Aspects of the Finite Element Method. Lecture Notes in Mathematics, 606, Springer-Verlag, Berlin. https://doi.org/10.1007/BFb0064470

Rui, H. X., \& Pan, H. (2012). A block-centered finite difference method for the Darcy-Forchheimer model. SIAM J. Numer. Anal., 50(5), 2612-2631. https://doi.org/10.1137/110858239

Russell, T. F. (1995). Rigorous block-centered discretization on irregular grids: Improved simulation of complex reservoir systems. Project Report, Research Corporation, Tulsa.

Shen, P. P., Liu, M. X., \& Tang, L. (2002). Mathematical model of petroleum exploration and development. Science Press, Beijing.

Sun, T. J., \& Yuan, Y. R. (2009). An approximation of incompressible miscible displacement in porous media by mixed finite element method and characteristics-mixed finite element method. J. Comput. Appl. Math., 228(1), 391-411. https://doi.org/10.1016/j.cam.2008.09.029

Todd, M. R., O’Dell, P. M., \& Hirasaki, G. J. (1972). Methods for increased accuracy in numerical reservoir simulators. Soc. Petrol. Engry. J., 12(6), 521-530. https://doi.org/10.2118/3516-PA

Vogt, C. H. (1982). A homogenization theorem leading to a Volterra integro-differential equation for permeation chromotography. SFB 123, Preprint 155, Heidelberg, SFB-Preprints.

Weiser, A., \& Wheeler, M. F. (1988). On convergence of block-centered finite difference for elliptic problems. SIAM J Numer. Anal., 25(2), 351-375. https://doi.org/10.1137/0725025

Yang, D. P. (2000). Analysis of least-squares mixed finite element methods for nonlinear nonstationary convectionCdiffusion problems. Math. Comp., 69(231), 929-963.

Yuan, Y. R. (1996). Characteristic finite element methods for positive semidefinite problem of two phase miscible flow in three dimensions. Chin. Sci. Bull., 41(22), 2027-2032.

Yuan, Y. R. (1999). Characteristic finite difference methods for positive semidefinite problem of two phase miscible flow in porous media. J. Systems Sci. Math. Sci., 12(4), 299-306.

Yuan, Y. R. (2013). Theory and application of reservoir numerical simulation. Science Press, Beijing.

\section{Copyrights}

Copyright for this article is retained by the author(s), with first publication rights granted to the journal.

This is an open-access article distributed under the terms and conditions of the Creative Commons Attribution license (http://creativecommons.org/licenses/by/4.0/). 